\title{
PRIMEIRO REGISTRO DE PEGADAS DE MESOSAURIDAE (AMNIOTA, SAUROPSIDA) NA FORMAÇÃO IRATI (PERMIANO SUPERIOR DA BACIA DO PARANÁ) DO ESTADO DE GOIÁS, BRASIL
}

\author{
FERNANDO ANTONIO SEDOR \\ Museu de Ciências Naturais, Setor de Ciências Biológicas, UFPR, Centro Politécnico, Jardim das Américas, \\ 81531-990, Cx. P. 19031, Curitiba, PR, Brasil. sedor@ufpr.br \\ RAFAEL COSTADA SILVA \\ Museu Nacional, DGP, UFRJ, Quinta da Boa Vista, São Cristóvão, 20940-040, Rio de Janeiro, RJ, Brasil. \\ paleoicno@hotmail.com
}

\begin{abstract}
RESUMO - Pesquisas recentes revelaram a presença de icnofósseis de Mesosauridae Baur, 1889 em cinco amostras de calcário laminar de coloração branca a cinza da Formação Irati nos municípios de Perolândia e Portelândia, sul do Estado de Goiás. Preservadas em hiporrelevo convexo, as pegadas constituem-se de dois a quatro traços paralelos ou ligeiramente divergentes com espaçamento regular e curvados em um mesmo sentido; a porção anterior de cada traço geralmente é alargada e encurvada com a extremidade anterior arredondada ou rombuda; a porção posterior é afilada e com extremidade aguda. Estas características ocorrem em pegadas encontradas no Paraná, interpretadas como marcas de arraste de dedos dos pés produzidas por representantes dos Mesosauridae em natação, não constituindo uma impressão do autopódio. As pegadas representam provavelmente a impressão das extremidades dos dedos II, III, IV, e eventualmente do dedo I. A largura dos dígitos e o espaçamento entre os dedos conferem com a morfologia e as dimensões dos apêndices locomotores dos Mesosauridae. Esta ocorrência consiste no primeiro registro de pegadas fósseis no Estado de Goiás, além de constituir a segunda localidade conhecida de ocorrência de icnofósseis atribuídos a Mesosauridae em todo o "Sistema Irati-Whitehill".
\end{abstract}

Palavras-chave: icnofósseis, Proganosauria, Mesosauridae, bacia do Paraná, Formação Irati, Permiano, América do Sul.

ABSTRACT - FIRST MESOSAURIDAE (AMNIOTA, SAUROPSIDA) FOOTPRINTS IN IRATI FORMATION (UPPER PERMIAN, PARANÁ BASIN), BRAZIL. Recent studies revealed the presence of Mesosauridae traces at Perolândia and Portelândia localities (southern Goiás). Five samples of white to gray laminar limestone, bearing the ichnofossils, preserved in convex hyporelief. The footprints show two to four parallel or slightly divergent traces with regular spacing, and bent in the same direction. The foremost portion of trace is generally enlarged and curved, with rounded or stumped edge. The back portion is relatively thinner, and with pointed edge. The same characteristics are shown by prints found in Paraná State, which were interpreted as drag marks of the fingers produced by swimming mesosaurs. Therefore, they are not autopodium impressions. The prints most likely represent the impressions left by digits II, III, IV, and eventually, from digit I. The width of the traces, as well as the spacing between them agree with morphology and dimensions recorded before for Mesosauridae autopodium. This is the first tetrapod traces recorded at Goiás State, besides being the second acknowledged occurrence of ichnofossils attributed to Mesosauridae in the whole "Irati-Whitehill System".

Key words: ichnofossils, Proganosauria, Mesosauridae, Paraná basin, Irati Formation, Permian, South America.

\section{INTRODUÇÃO}

A família Mesosauridae Baur, 1889 reúne formas extintas de animais lacertóides, esguios e de pequeno porte, que raramente ultrapassam um metro de comprimento. Estes são os mais antigos amniotas conhecidos com adaptações à vida ao ambiente aquático, tais como corpo longo e delgado, cauda longa e comprimida lateralmente, crânio com um longo rostro, narinas situadas a frente das órbitas, grandes cavidades orbitárias, anel esclerótico, costelas paquiostóticas, autopódios com dedos lon- gos e membranas interdigitais, etc (Cope, 1886; McGregor, 1908; Williston, 1914; Romer, 1966; Rösler \& Tatizana, 1985; Carroll, 1988). Provavelmente eram nadadores ágeis e capazes de realizar manobras rápidas dentro d'água (Williston, 1914).

No continente africano, os Mesosauridae ocorrem na Formação Whitehill do Sistema Karoo e, na América do Sul, são conhecidas ocorrências para o Brasil, Paraguai e Uruguai. No Brasil, os Mesosauridae foram registrados na Formação Irati (Permiano Superior da bacia do Paraná) nos estados do Rio Grande do Sul, Santa Catarina, Paraná, São Paulo, 
Goiás e Mato Grosso (e.g. McGregor, 1908; Shikama \& Ozaki, 1966; Mendes, 1967; Araújo, 1976; Borgomanero \& Leonardi, 1979; Moreira et al., 1984).

Apesar da ampla distribuição das formações Irati e Whitehill e de restos de mesossaurídeos serem relativamente abundantes em rochas de ambos os continentes, são ainda raras as ocorrências de icnofósseis produzidos por estes animais. As primeiras pegadas atribuídas a Mesosauridae foram registradas no município de Guapirama, norte do Estado do Paraná, em rochas da Formação Irati (Sedor et al., 2001), mas não foram ilustradas pelos autores na ocasião. O presente estudo revela a presença de estruturas similares na mesma formação nos municípios de Perolândia e Portelândia, sul do Estado de Goiás.

\section{CONTEXTOGEOLÓGICO}

A Formação Irati aflora dos estados do Rio Grande do Sul a Goiás e é constituída por folhelhos e argilitos cinzaescuros, folhelhos pirobetuminosos, arenitos, margas e calcários associados, sobrepostos à Formação Palermo e sotopostos às formações Serra Alta e Corumbataí (Schneider et al., 1974). A principal estrutura sedimentar é a laminação plano-paralela, mas nos estratos carbonáticos são observados localmente marcas onduladas, laminação cruzada, oólitos, brechas intraformacionais e laminação algálica (Schneider $e t$ al., 1974). Ocorrem também estratificações cruzadas hummocky e estruturas wave (Lavina, 1991).

Esta formação constitui a base da seqüência permiana superior e representa um extenso mar epicontinental, progressivamente mais salino da base para o topo, que cobriu o sul do Gondwana; é correlata à Formação Whitehill da bacia do Karoo, ao sul do continente africano, e à Formação Mangrullo, no Uruguai. Há discordância entre os autores em relação às idades atribuídas paras as unidades do "Sistema Irati-Whitehill". As rochas africanas foram datadas entre o Permiano Inferior e Permiano Médio (Pinto, 1972; Oelofsen \& Araújo, 1987), correspondendo à porção mais superior do Sakmariano, ao passo que as rochas sul-americanas representam o intervalo temporal entre Kazaniano/Tatariano (Daemon \& Quadros, 1970).

A faciologia apresentada pela formação reflete uma complexa geometria de bacia durante a fase de deposição, quando esta era constituída por golfos e baías de profundidades e salinidades variáveis em condições de um mar restrito (Milani et al., 1994).

\section{MATERIAL}

O material de estudo consiste de cinco amostras (MCN.P.262, MCN.P.266, MCN.P.426, MCN.P.427 e MCN.P.428) de calcário laminar de coloração branca a cinza, contendo icnofósseis preservados em hiporrelevo convexo. As amostras foram coletadas em 1998 nas pedreiras de calcário METAGO, Unidade I e II, Município de Portelândia, e SUCAL (17²9'00” S/ 5203'11,6" W), no município de Perolândia, ambos no Estado de Goiás (Figura 1). Também foi examinada a amostra MCN.P.293, procedente de Guapirama (PR) e ilustrada para comparação (Figura 2A). Todas as amostras estudadas encontram-se depositadas na coleção paleontológica do Museu de Ciências Naturais, Setor de Ciências Biológicas, Universidade Federal do Paraná (MCN-SCB-UFPR), Curitiba, Brasil.

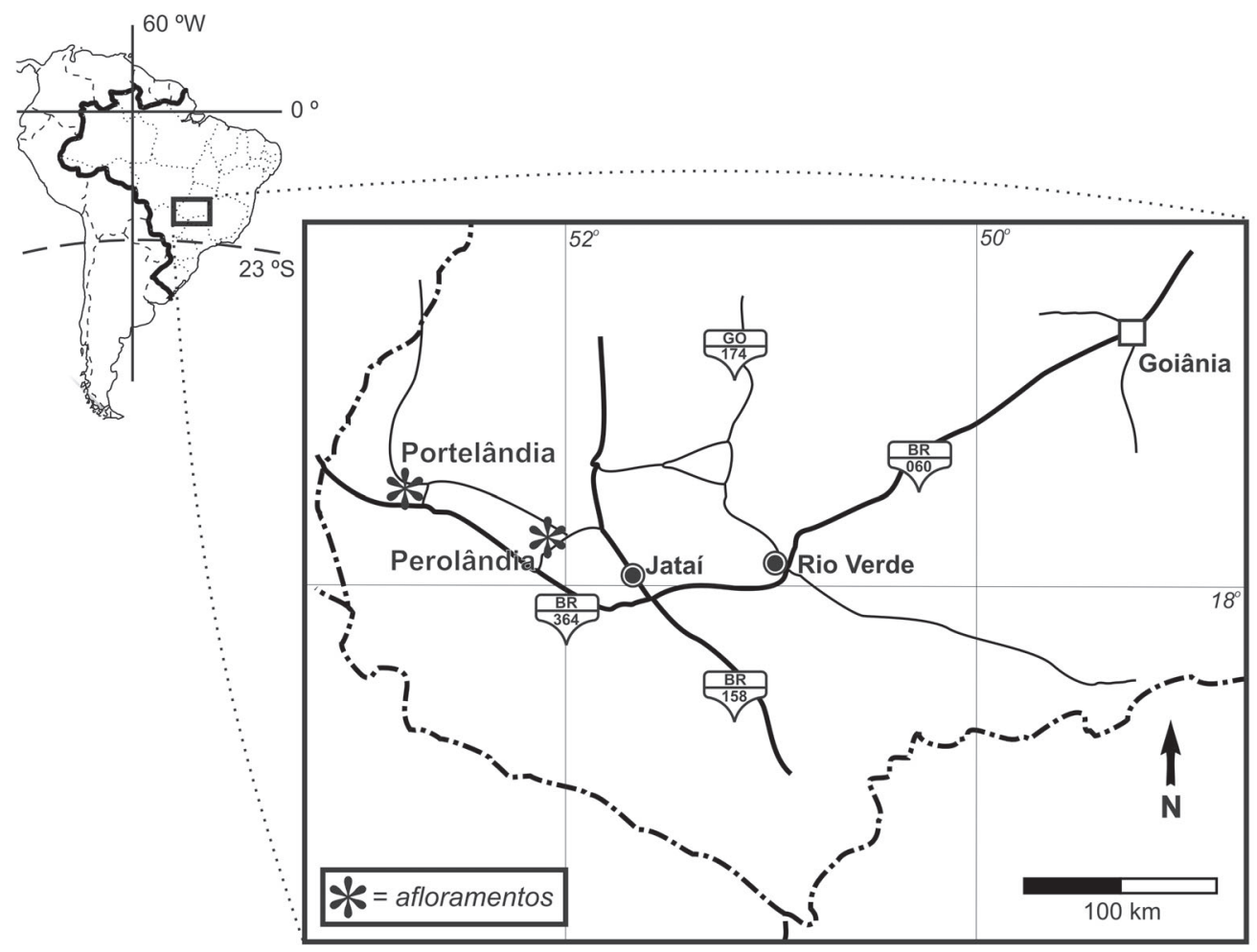

Figura 1. Mapa de localização dos afloramentos com ocorrências de pegadas fósseis no Estado de Goiás. Figure 1. Location map of the outcrops with Mesosauridae footprints occurrences in Goiás State. 


\section{ICNOLOGIA}

Os icnofósseis procedentes do município de Guapirama foram descritas por Sedor et al. $(2001,2002)$ como pegadas constituídas por dois ou três traços aproximadamente paralelos e ligeiramente curvados, podendo ocorrer uma quarta marca de arraste de menores dimensões no lado interno; a extremidade anterior é mais profunda e fortemente encurvada medialmente em forma de vírgula (Figuras 2B, 2C e 2D). Foram interpretadas pelos autores como marcas de arraste dos dígitos II, III, IV e eventualmente I dos pés de mesossaurídeos em natação.
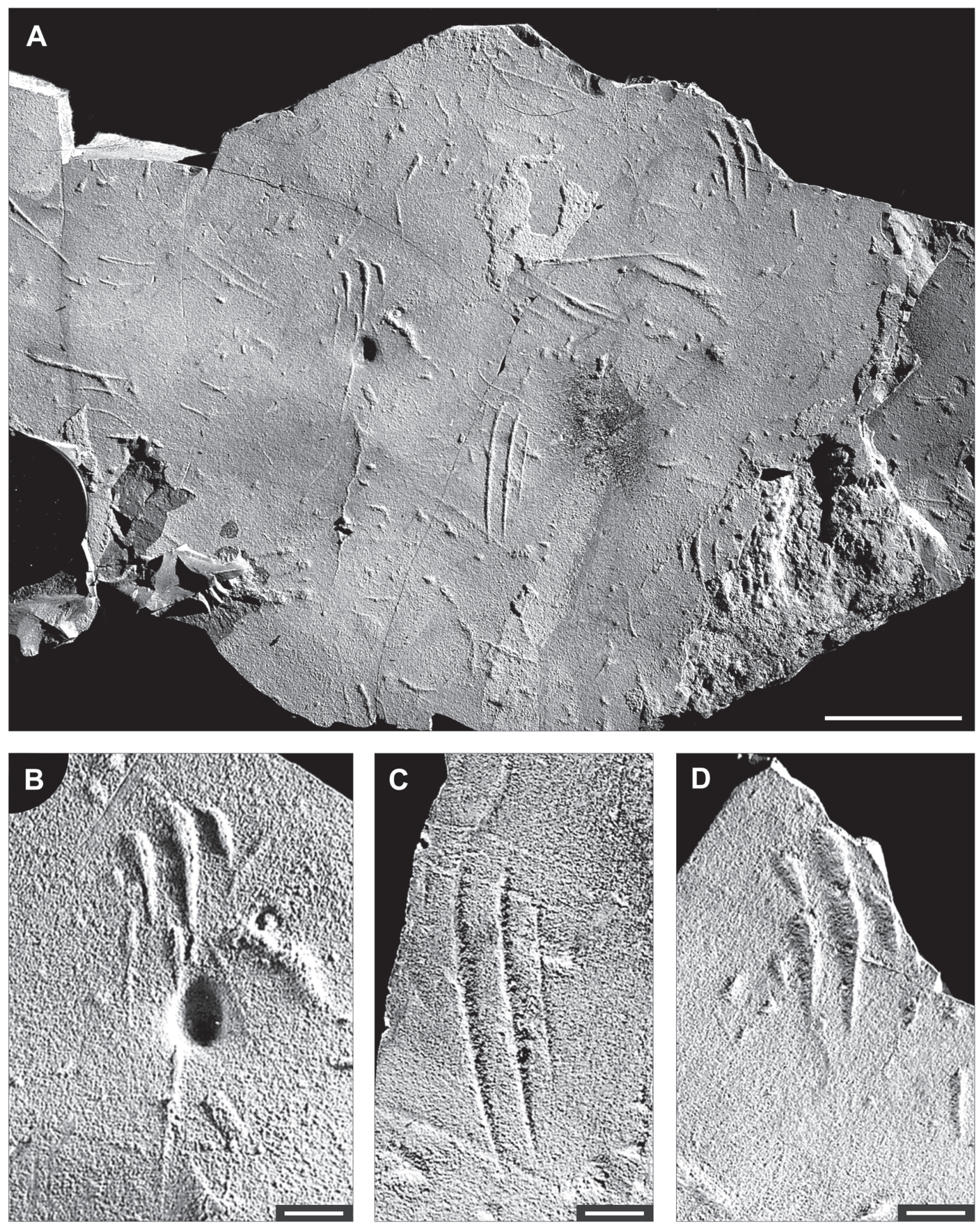

Figura 2. Pegadas de Mesosauridae procedentes de Guapirama, Estado do Paraná (MCN.P.293). A. Vista geral da amostra, escala $=5 \mathrm{~cm}$. B-D. Detalhes da mesma amostra. Escala $=1 \mathrm{~cm}$.

Figure 2. Mesosauridae footprints from Guapirama, Paraná State (MCN.P.293). A. General view, scale bar $=5 \mathrm{~cm}$. B-D. Detailed view. Scale bar $=1 \mathrm{~cm}$. 
Dentre o material estudado (Figura 3) ocorrem icnofósseis constituídos por dois a quatro traços paralelos (Figuras $3 \mathrm{~A} \mathrm{e}$ $3 \mathrm{E}$ ) ou ligeiramente divergentes (Figuras 3B, 3C e 3E) com espaçamento regular e curvados em um mesmo sentido. A porção anterior de cada traço geralmente é alargada e encurvada com a extremidade anterior arredondada ou rombuda, e a porção posterior é afilada e com extremidade aguda. Associados às pegadas ocorrem também alguns traços de dígitos isolados e traços duplos sinuosos (Figuras 3B e 3C). As pegadas ocorrem isoladamente e não formam pistas.
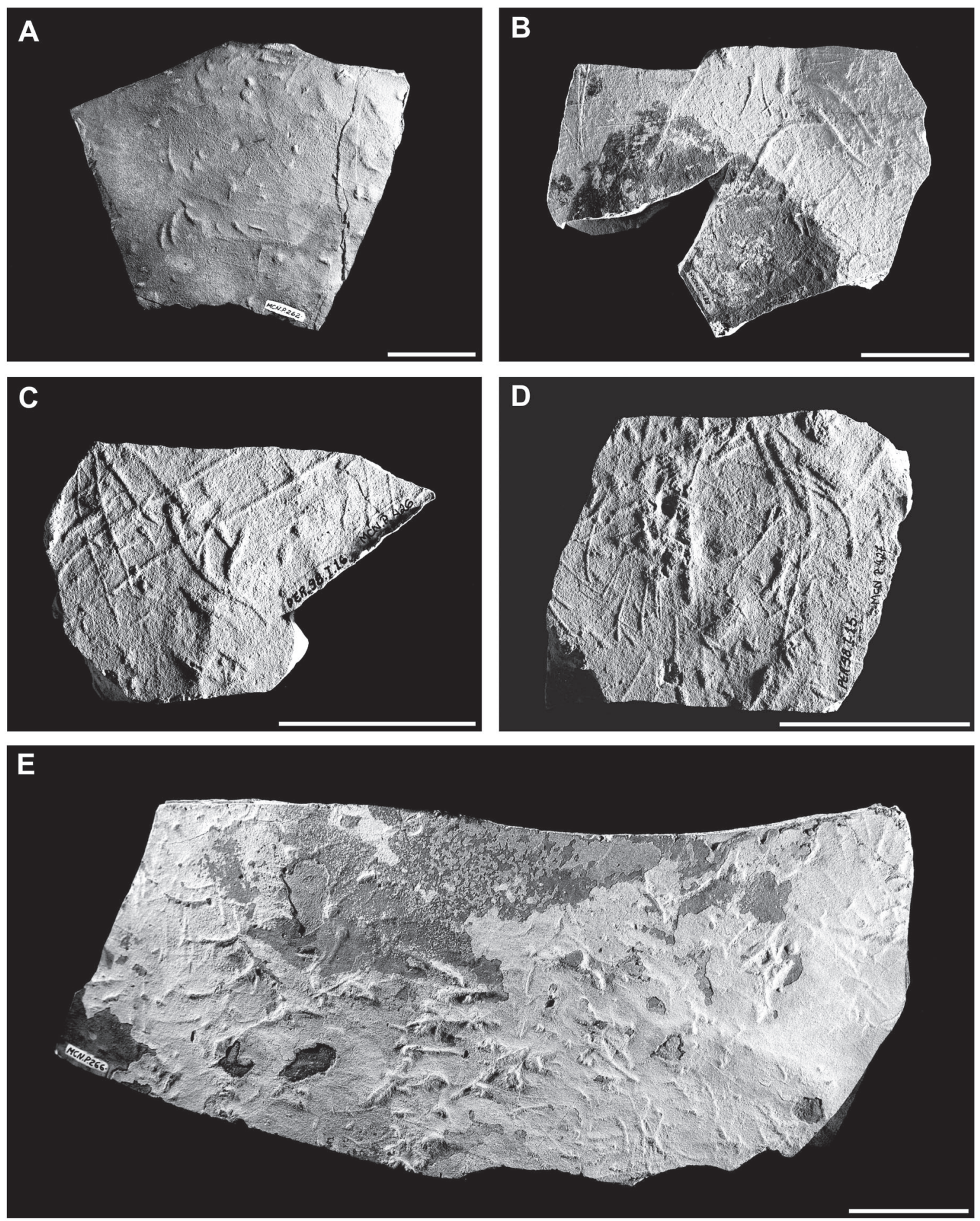

Figura 3. Pegadas de Mesosauridae procedentes de Portelândia e Perolândia, Estado de Goiás. A. MCN.P.262. B. MCN.P.428. C. MCN.P.426. D. MCN.P.427. E. MCN.P.266. Escala $=5 \mathrm{~cm}$.

Figure 3. Mesosauridae footprints from Portelândia and Perolândia, Goiás State. A. MCN.P.262. B. MCN.P.428. C. MCN.P.426. D. MCN.P.427. E. MCN.P.266. Scale bar $=5 \mathrm{~cm}$. 


\section{DISCUSSÃO}

Além das conhecidas adequações ao hábito aquático presentes entre as espécies de Mesosauridae (Cope, 1886; McGregor, 1908; Williston, 1914; Romer, 1966; Rösler \& Tatizana, 1985; Carroll, 1988), estes constituem os únicos tetrápodes conhecidos em todo o "Sistema Irati-Whitehill”, tornando possível a ocorrência de pegadas e pistas destes animais em sedimentos de ambientes subaquáticos.

Os apêndices locomotores posteriores dos Mesosauridae são proporcionalmente maiores que os anteriores, o autopódio posterior apresenta quase o dobro das dimensões do autopódio anterior, sendo aplanado e alargado. Os dedos dos pés dos mesossaurídeos eram longos e interligados por uma membrana interdigital como uma nadadeira (McGregor, 1908; Williston, 1914; Romer, 1956; Borgomanero \& Leonardi, 1979; Rösler \& Tatizana, 1985). A forma dos autopódios posteriores dos Mesosauridae e seu alinhamento com o eixo do corpo (Williston, 1914; Sedor \& Ferigolo, 1999) favoreceriam que as extremidades dos dígitos II, III, IV, e eventualmente do dedo I, tocassem o substrato. Os Mesosauridae apresentavam uma longa cauda comprimida lateralmente (McGregor, 1908; Sedor, 1994) que provavelmente era o principal apêndice propulsor na água.

Pegadas fósseis produzidas em ambiente subaquático foram estudadas por McAllister (1989), que definiu critérios para o reconhecimento dessas estruturas, tais como alongamento e curvatura dos traços e impressão preferencial das extremidades distais dos dedos, grande variação de ângulo do passo, comprimento dos traços excessivamente variável em relação à largura e configurações aleatórias de pegadas. Essas são características presentes nas pegadas procedentes da Formação Irati, o que juntamente com a interpretação de paleoambiente marinho para a Formação Irati reforça sua origem subaquática.

Dessa forma, as pegadas encontradas na Formação Irati nos estados de Goiás e Paraná podem ser interpretadas como marcas de arraste das extremidades dos dedos dos pés produzidas por representantes dos Mesosauridae em natação, e assim não constituem uma impressão do autopódio. Os icnofósseis foram produzidos durante o deslocamento subaquático do animal próximo ao fundo inconsolidado com as extremidades dos dedos dos membros locomotores posteriores, mais longos que os anteriores. As pegadas representam provavelmente a impressão das extremidades dos dedos II, III, IV e eventualmente do dedo I. A largura dos dígitos e o espaçamento entre os dedos conferem com a morfologia e as dimensões dos apêndices locomotores dos Mesosauridae (McGregor, 1908; Romer, 1956; Borgomanero \& Leonardi, 1979; Sedor, 1994). As pegadas divergentes (Figuras 3A e 3D) provavelmente foram produzidas pelos apêndices locomotores posteriores durante os impulsos para aumento de velocidade e/ou mudança de curso, quando a resistência água inflava as membranas interdigitais promovendo uma abertura dos ângulos dos dedos. As pegadas com traços paralelos extremamente alongados (Figura 3C) provavel- mente foram produzidas pelo arraste das extremidades dos dedos sobre o sedimento de fundo durante movimentos de "inércia" ou os proporcionados unicamente pelas ondulações da longa cauda.

\section{CONCLUSÕES}

Os icnofósseis estudados correspondem a marcas de arraste de dedos dos pés produzidas por mesossaurídeos durante o deslocamento subaquático próximo ao fundo inconsolidado. Pegadas divergentes foram produzidas durante os impulsos para aumento de velocidade e/ou mudança de curso, enquanto as membranas interdigitais eram infladas, e traços paralelos extremamente alongados correspondem ao arraste dos dedos sobre o fundo durante movimentos de "inércia" ou de propulsão pela cauda.

Esta ocorrência consiste no primeiro registro de pegadas fósseis de tetrápodes no Estado de Goiás, além de constituir a segunda localidade conhecida de ocorrência de icnofósseis atribuídos a Mesosauridae em todo o "Sistema IratiWhitehill", fornecendo novas evidências do hábito aquático entre os Mesosauridae e criam novas expectativas para a pesquisa de icnofósseis permianos no Brasil.

\section{AGRADECIMENTOS}

Os autores expressam seus agradecimentos ao $\mathrm{Mu}$ seu de Ciências Naturais (MCN-SCB-UFPR) e à Direção do Setor de Ciências Biológicas da Universidade Federal do Paraná pelo apoio e pelas condições oferecidas para o desenvolvimento deste trabalho. Agradecem também a Ricardo Queiroz pela colaboração no texto em língua inglesa, e a Sibelle Trevisan Disaró e Antonio Carlos Sequeira Fernandes pelas valiosas sugestões no manuscrito.

\section{REFERÊNCIAS}

Araújo, D.C. 1976. Taxonomia e relações dos Proganosauria da bacia do Paraná. Anais da Academia Brasileira de Ciências, 48(1):91-116.

Borgomanero, G. \& Leonardi, G. 1979. Anel esclerótico e outras peculiaridades em um espécime de Stereosternum tumidum (Proganosauria, BAUR, 1887) de Assistência, São Paulo. In: SIMPÓSIO REGIONAL DE GEOLOGIA, 2, 1979. Atas, Rio Claro, SBG, 1:175-179.

Carroll, R.L. 1988. Vertebrate Paleontology and Evolution. New York, W.H. Freeman and Company, 566 p.

Cope, E.D. 1886. A contribution to the vertebrate paleontology of Brazil. Proceedings of the American Philosophical Society, 23:715 .

Daemon, R.F. \& Quadros, L.P. 1970. Bioestratigrafia do Neopaleozóico da bacia do Paraná. In: CONGRESSO BRASILEIRO DE GEOLOGIA, 24, 1970. Anais, Brasília, SBG, p. 355-412.

Lavina, E.L. 1991. Geologia sedimentar e paleogeografia do Neopermiano e Eotriássico (Intervalo Kazaniano - Scythiano) da bacia do Paraná. Programa de Pós-Graduação em Geociências, Universidade Federal do Rio Grande do Sul, Tese de Doutorado, 333 p. 
McAllister, J. 1989. Dakota Formation tracks from Kansas: implications for the recognition of tetrapod subaqueous traces. In: D.D. Gillette \& M.G. Lockley (eds.) Dinosaur Tracks and Traces, Cambridge Universiy Press, p. 343-348.

McGregor, J.H. 1908. Mesosaurus brasiliensis nov. sp. In: Relatório Final Comissão de Estudos Minas de Carvão de Pedra do Brasil, Rio de Janeiro, v.2, p. 301-336.

Mendes, J.C. 1967. The Passa Dois Group (The Brazilian portion of the Paraná Basin). In: J.J. Bigarella; R.D. Becker \& I.D. Pinto (eds.) Problems in Brazilian and Gondwana Geology, Centro de Investigações do Gondwana (UFRGS)/Instituto de Geologia (UFPR), p. 119-166.

Milani, E.J.; França, A.B. \& Schneider, R.L. 1994. Bacia do Paraná. Boletim de Geociências da Petrobrás, 8(11):69-82.

Moreira, L.E.; Ribeiro, M.B. \& Lima, B.C. 1984. Mesossaurídeos em Goiás. Anuário de Divulgação Científica da Universidade Católica de Goiás, 10:125-133.

Oelofsen, B. \& Araújo, D.C. 1987. Mesosaurus tenuidens and Stereosternum tumidum from the Permian Gondwana of both Southern Africa and South America. South African Journal of Science, 83:370-372.

Pinto, I.D. 1972. Late paleozoic insects and crustaceans from Paraná basin and their bearing on chronology and continental drift. Anais da Academia Brasileira de Ciências, 44:247-257 (Suplemento).

Romer, A.S. 1956. Osteology of the Reptiles. Chicago, Chicago University Press, $772 \mathrm{p}$.

Romer, A.S. 1966. Vertebrate Paleontology. Chicago, Chicago University Press, $468 \mathrm{p}$.

Rösler, O. \& Tatizana, C. 1985. As membranas natatórias em Stereosternum tumidum. Coletânea de Trabalhos Paleontológicos, Brasília, DNPM, p. 129-131 (Série Geológica, 27/Série Paleontologia e Estratigrafia, 2).
Schneider, R.L.; Mühlmann, H.; Tommasi, E.; Medeiros, R.A.; Daemon, R.F. \& Nogueira, A.A. 1974. Revisão Estratigráfica da bacia do Paraná. In: CONGRESSO BRASILEIRO DE GEOLOGIA, 28, 1974. Anais, Porto Alegre, SBG, 1:41-66.

Sedor, F.A. 1994. Estudo pós-craniano de Brazilosaurus sampauloensis Shikama \& Ozaki, 1966 (Anapsida, Proganosauria, Mesosauridae) da Formação Irati, Permiano da bacia do Paraná, Brasil. Programa de Pós-graduação em Geociências, Universidade Federal do Rio Grande do Sul, Dissertação de Mestrado, $91 \mathrm{p}$.

Sedor, F.A. \& Ferigolo, J. 1999. Apêndices locomotores escapulares e pélvicos em Brazilosaurus sanpauloensis Shikama \& Ozaki, 1966 (Proganosauria, Mesosauridae), Formação Irati, Permiano da bacia do Paraná, Brasil. In: CONGRESSO BRASILEIRO DE PALEONTOLOGIA, 16, 1999. Resumos, Crato, SBP, p. 104.

Sedor, F.A.; Costa. R. \& Leonardi, G. 2001. Icnofósseis de Mesosauridae (Proganosauria) na Formação Irati (Neopermiano da bacia do Paraná), Estado do Paraná, Brasil. In: CONGRESSO BRASILEIRO DE PALEONTOLOGIA, 17, 2001. Resumos, Rio Branco, SBP, p. 22.

Sedor, F.A.; Costa. R. \& Leonardi, G. 2002. Upper Permian Tetrapoda footprints from the Irati and Rio do Rasto formations, Paraná Basin, Brazil. Journal of Vertebrate Paleontology, 22(supplement to number 3):106A.

Shikama, T. \& Ozaki, H. 1966. On a reptilian skeleton from the Paleozoic formation of San Paulo, Brazil. Transaction Proceedings of Paleontological Society of Japan, 64:351358.

Williston, S.W. 1914. Water Reptiles of The Past and Present. Chicago, University Chicago Press, $251 \mathrm{p}$. 